\title{
Hodgkin's Lymphoma Presenting as Multiple Cavitary Lung Lesions
}

\author{
Esha Jain ${ }^{1}$, Ali Hani Al-Tarbsheh ${ }^{1}$, Jozef Oweis ${ }^{1}$, Erik Jacobson ${ }^{2}$, Boris Shkolnik ${ }^{3}$ \\ ${ }^{1}$ Department of Medicine, Albany Medical Center, Albany, New York, USA \\ 2 Department of Radiology, Albany Medical Center, Albany, New York, USA \\ ${ }^{3}$ Department of Medicine, Division of Pulmonary and Critical Care Medicine, Albany Medical Center, Albany, New York, USA
}

Received: 23/10/2021

Accepted: 08/11/2021

Published: 01/12/2021

How to cite this article: Jain E, Al-Tarbsheh AH, Oweis J, Jacobson E, Shkolnik B. Hodgking's lymphoma presenti as a multiple cavitary lung lesion. EJCRIM 2021;8: doi:10.12890/2021_003024.

Conflicts of Interests: The authors declare there are no competing interests.

Acknowledgements: Ali Al-Tarbsheh and Esha Jain are first authors as they contributed equally to this case.

This article is licensed under a Commons Attribution Non-Commercial 4.0 License

\section{ABSTRACT}

Hodgkin Lymphoma (HL) typically presents similarly to an infectious etiology, thus awareness of its atypical presentations is essential. We present a case of an adult woman who was found to have $\mathrm{HL}$ after presenting with a dry, non-productive cough and showing cavitary lesions on chest computed tomography (CT). We also describe the clinical, laboratory, and radiological workup done leading to the diagnosis and management of $\mathrm{HL}$ in a critical care setting.

\section{LEARNING POINTS}

- Cavitary lung lesions, particularly multiloculated, are often caused by mycobacterium tuberculosis (TB), aspergillosis, granulomatosis with polyangiitis, sarcoidosis, and rheumatic nodules.

- Pulmonary infiltration is a rare disorder of an extra-nodal site in Hodgkin's Lymphoma. The mediastinum and head and neck regions remain the most common sites affected by HL.

- $\quad$ Radiologically, primary pulmonary HL may mimic pneumonia, carcinoma making the diagnosis unclear.

\section{KEYWORDS}

Cavitory lung lesions, lymphoma, Hodgkin's lymphoma, primary lung lymphoma

\section{INTRODUCTION}

Hodgkin Lymphoma (HL) with pulmonary involvement is much less commonly observed than classic HL. About 15-40\% of cases of Hodgkin's lymphoma include pulmonary involvement ${ }^{[1]}$. Pulmonary manifestations are most commonly observed through secondary involvement, while primary pulmonary Hodgkin lymphoma is relatively rare. Typically, HL can present as a single nodule, multiple nodules, or a mass. Although cavitary lesions can result from chemotherapy, multiple cavitary lesions are extremely uncommon at the time of diagnosis ${ }^{[2]}$ We present a case of a young adult woman who presented with a chronic dry cough and was found to have HL, nodular sclerosing subtype.

\section{CASE DESCRIPTION}

A 26-year-old African American woman with a past medical history of asthma, allergic rhinitis, and infectious mononucleosis presented with a 3-month history of a dry, non-productive cough. Her symptoms began while traveling from Albany, New York to New Orleans Louisiana for work. She returned to the northeast about 1.5 months before presenting to Albany Medical Center with persisting symptoms. Upon her return, she noted a lump in her left side of the neck that had been stable in size but was painful upon coughing. In a questionnaire she reported night sweats and negative for fever, chills, chest pain, shortness of breath, or weight loss. 
The patient denied any history of tobacco use, incarceration, or homelessness. She noted no recent contact with sick persons.

Blood pressure on admission was 114/88 mm Hg, heart rate was 95 beats/min, respiratory rate was $16 / \mathrm{min}$, and temperature was $36.8^{\circ} \mathrm{C}$. Her oxygen saturation was $97 \%$ in room air. Physical examination revealed immobile, hard, and non-tender $2 \times 2 \mathrm{~cm}$ left supraclavicular lymph nodes on palpation.

Laboratory testing resulted in a complete blood count with a hemoglobin level of $10.4 \mathrm{gm} / \mathrm{dl}$, a hematocrit level of $34.4 \%$, a white blood cell count of $9,000 / \mathrm{m3}$, a platelet count of $492,000 / \mathrm{mm}^{3}$, and MCV $91.5 \mathrm{fL}$. Automated differential blood test showed neutrophils $61 \%$, lymphocytes $21 \%$, monocytes $8 \%$, and eosinophils $9 \%$ (absolute eosinophils $0.8 \%$ ). The complete metabolic panel disclosed electrolytes within normal limits, albumin elevated measuring $3.3 \mathrm{gm} / \mathrm{dL}$, and lactose dehydrogenase (LDH) elevated measuring 192.

The patient underwent an infectious and malignancy workup involving radiographic imaging, cultures, and serology. Chest CT revealed a large mediastinal mass $(12 \times 7 \times 8 \mathrm{~cm})$ infiltrating the medial left upper lobe with regions of hypodensity (Fig. 1). Chest CT also revealed multiple bilateral cavitary lung nodules and masses of varying sizes, predominantly in the upper lobes. A single nodule was observed in the right upper lobe (Fig. 2). The largest lobulated mass measured about $8 \times 6 \times 4 \mathrm{~cm}$. Additionally, radiography revealed enlarged mediastinal and left supraclavicular lymph nodes.
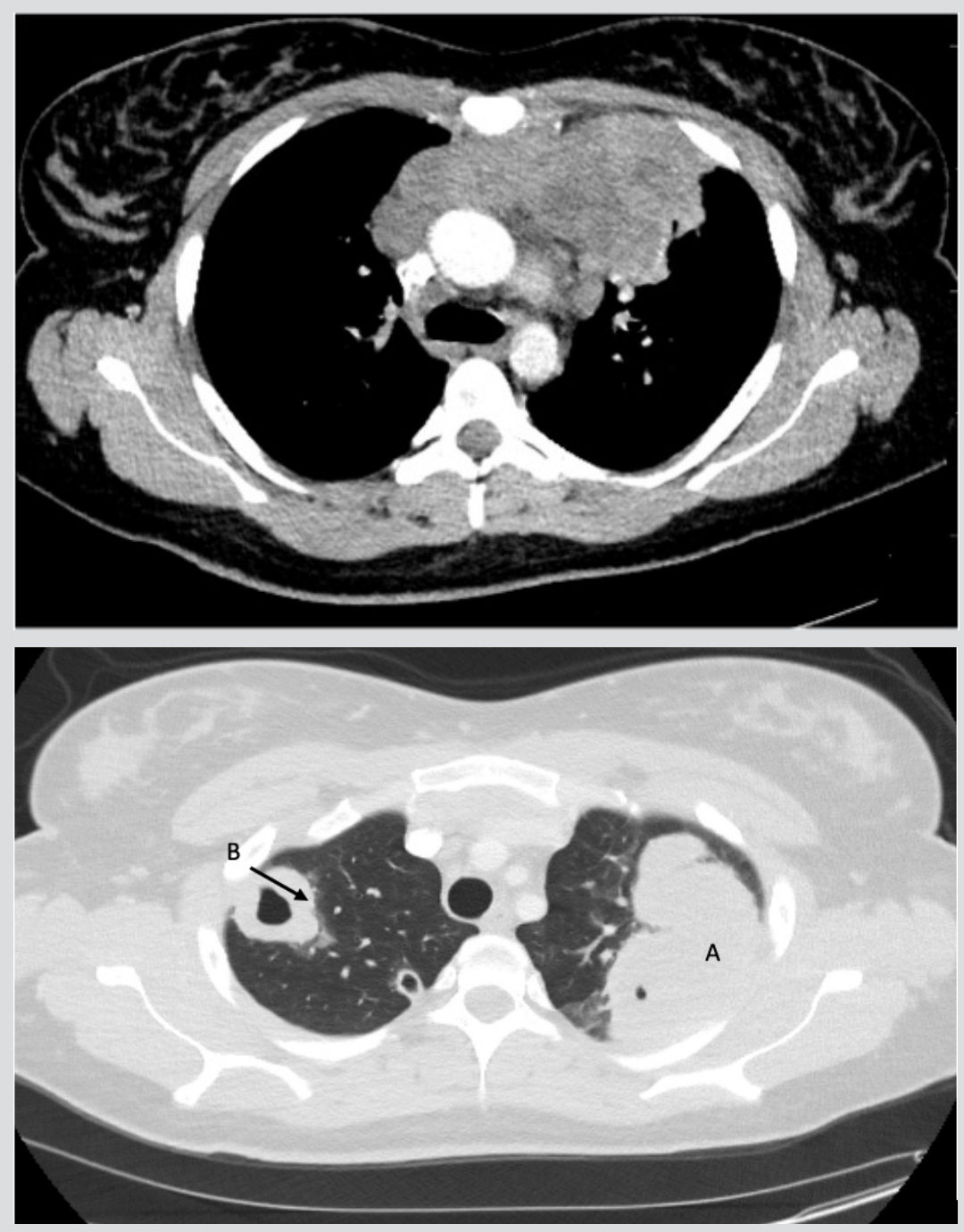

Figure 1. Chest CT revealed large lobular anterior mediastinal mass with areas of central hypodensity, likely necrosis.

Figure 2. CT scan revealed revealed a large soft tissue mass in the left upper lobe (A). Thick-walled cavitary lesions are noted in the right upper lobe (B)

Infectious workup was negative for acid-fast bacterial (AFB) cultures, histoplasmosis, aspergillus, serum quantiferon, fungitell assay, human immunodeficiency virus (HIV), and SARS CoV-2. Serology showed negative EBV IgM, positive EBV IgG, EBV early antigen, EBV nuclear antigen, and a negative EBV by PCR. 
Subsequently, excisional lymph node biopsy and bronchoscopy with bronchobiliary lavage was performed. The pathology report from the left neck mass confirmed classic Hodgkin lymphoma, nodular sclerosis type with bronchopulmonary lavage revealed benign bronchial cells. The hematology and oncology service was consulted and recommended outpatient initiation of a chemotherapy regimen. At six month follow-up after discharge, she was doing well on her current chemotherapy regimen, pending a follow-up PET scan.

\section{DISCUSSION}

Hodgkin's Lymphoma (HL) is a lymphoid neoplasm that originates from B lymphocytes and involves few malignant cells mixed among a heterogeneous population of non-neoplastic inflammatory cells. HL has been divided into classic Hodgkin's lymphoma (CHL) and nodular lymphocyte-predominant Hodgkin's lymphoma (NLPHL). CHL makes up about $90 \%$ of all HL cases and is subdivided into four categories: nodular sclerosis (NSCHL), mixed cellularity (MCCHL), lymphocyte rich (LRCHL), and lymphocyte depleted (LDCHL) ${ }^{[3]}$. Of these, NSCHL is the most common ${ }^{[1]}$.

HL most often occurs in the mediastinum and head and neck regions, however under uncommon circumstances, HL may present at extranodal sites. Pulmonary infiltration has been noted to occur in about $15-40 \%$ of $\mathrm{HL}_{\text {cases }}{ }^{[1]}$. Typically, pulmonary infiltration appears to occur later during disease progression and is almost always associated with mediastinal or hilar node involvement ${ }^{[4]}$. Pulmonary involvement has been noted to occur in about $40 \%$ of cases later in the disease course and about $60 \%$ at autopsies in cases of relapse. Generally, isolated primary pulmonary manifestations, caused by a clonal proliferation of the lymphoid cell line, are a much rarer finding, with about 100 reported cases $^{[5,6]}$. Previous reports have demonstrated that the diagnosis of primary pulmonary Hodgkin's Lymphoma requires three criteria to be fulfilled: the disease should be restricted to the lung in which the neoplastic process arises, histological results consistent with Hodgkin's Lymphoma should be present, and other possible pathologic causes should be excluded ${ }^{[4]}$. The present case fulfilled the latter two of these criteria.

Among the reported cases of $\mathrm{HL}$ with pulmonary involvement, the typical manifestation involves singular or multiple nodules or masses and primarily involves the superior portion of the lung ${ }^{[2,7]}$. Cavitary lung lesions, particularly multiloculated, are often associated with alternative etiologies such as mycobacterium tuberculosis (TB), aspergillosis, granulomatosis with polyangiitis, sarcoidosis, and rheumatic nodules ${ }^{[8]}$. Radiologically, primary pulmonary $\mathrm{HL}$ may mimic pneumonia, carcinoma, making the diagnosis unclear ${ }^{[9]}$. Due to its rare occurrence with $\mathrm{cHL}$ and the more frequent presentation of cavitary lesions for the previously mentioned etiologies, cavitary lesions may not lead a clinician to consider $\mathrm{HL}$ as part of their differential diagnosis, especially with its nonspecific symptoms. Garg and colleagues reported on a 22-yearold patient who presented with a 6-month history of B-symptoms, such as weight lose, night sweats, loss of apatite, fever and general fatigabilty and a non-productive cough. She was subsequently found to have a cavitating pulmonary lesion that was diagnosed and treated as pulmonary tuberculosis with no improvement. After repeated extensive workups, the patient underwent a cervical lymph node biopsy, which revealed binucleated Reed-Sternberg-like cells and mononuclear Hodgkin's cells, resulting in her diagnosis of NSCHL ${ }^{[2]}$. In our case, the patient presented similarly and was diagnosed through excisional lymph node biopsy.

Few case reports exist regarding NSCHL presenting with cavitary lesions. The diagnosis can be challenging, especially with nonspecific symptoms at the time of presentation. However, despite its rarity, consideration should be made in the appropriate clinical context. This case report highlights the importance of using clinical, laboratory, and radiological workups to properly diagnose and manage this patient population.

\section{REFERENCES}

Cooksley N, Judge DJ, Brown J. Primary pulmonary Hodgkin's lymphoma and a review of the literature since 2006. BMJ Case Rep 2014;2014. doi: 10.1136/bcr-2014-204020. Garg A, Kumar A, Kashyap R, Lal H. Hodgkin's lymphoma with cavitating lung lesion mimicking tuberculosis: A rare presentation. Lung India 2019;36(4):363-365. Roddie C, Peggs K. HodgkiŽ s lymphoma. Medicine. 2009;37:208-211.

McElnay PJ, Pawade J, Chandratreya L, West D. Giant thoracic mass: an unusual presentation of primary pulmonary Hodgkin's lymphoma. BMJ Case Rep. 2013;2013.

Diederich S, Link TM, Zühlsdorf H, Steinmeyer E, Wormanns D, Heindel W. Pulmonary manifestations of Hodgkin's disease: radiographic and CT findings. Eur Radiol 2001;11(11):2295-2305.

SinhaA,PattiR,Singh P,SolomonW,KupferY.ADiagnostic surprise:primaryHodgkin'slymphoma of thelung.JInvestigMed High ImpactCaseRep.2017;5(4):2324709617734247. Aljehani Y, Al-Saif H, Al-Osail A, Al-Osail E. Multiloculated cavitary primary pulmonary Hodgkin lymphoma: case series. Case Rep Oncol 2018;11(1):90-97.

Parkar AP, Kandiah P. Differential diagnosis of cavitary lung lesions. J Belg Soc Radiol 2016;100(1):100.

El Hage H, Hossri S, Samra B, El-Sayegh D. Primary pulmonary hodgkin's lymphoma: a rare etiology of a cavitary lung mass. Cureus 2017;9(8):e1620. 